\title{
Detailed observation on expression dynamics of Polycomb group genes during rice early endosperm development in subspecies hybridization reveals their characteristics of parent-of-origin genes
}

Quan Kuang ${ }^{1}$, Yinghua Wang ${ }^{3}$ and Shisheng $\mathrm{Li}^{2^{*}}$ (i)

\begin{abstract}
Background: Parent-of-origin gene expression and its role in seed development have drown a great attention in recent years. Genome-wide analysis has identified hundreds of candidate imprinted genes, a major type of parentof-origin genes, in rice hybrid endosperms at the stage of 5 days after pollination (dap). However, the expression of these genes in early endosperm have been never confirmed due to technique limitations and the behavior of the imprinted genes in different rice hybridizations are still largely unknown.

Results: Here, based on our elaborate technique established previously, the expression patterns of PcG genes in the early stages of endosperm development (within 3 dap), were comprehensively analyzed. We revealed that the free nucleus stage of endosperm development is critical for parent-of-origin gene analysis. The expression of the imprinted genes are highly dynamic, likely corresponding to the critical developmental events during this period. Hybridizations between Oryza sativa japonica and indica showed that the expression patterns of the same imprinted gene could be varied by crossing with different parental cultivars, indicative of their parent-dependent character. There are strong alleles that often showed predominant expression over other alleles regardless of the parental origin, which provides a possible explanation for the cultivar-dependent predominant phenotype in crop hybridizations. In addition, we found that the transcripts of the same gene behave differently, with imprinting or non-imprinting patterns, suggesting the existence of not only imprinted and non-imprinted genes but also imprinted or non-imprinted transcripts, which reveals new aspects of the genomic imprinting.
\end{abstract}

Conclusions: These findings on the characters of parent-of-origin genes shed light on the understanding the real role of gene imprinting in endosperm development.

Keywords: Polycomb group genes, Rice, Imprinting gene

\footnotetext{
* Correspondence: lishisheng2002@whu.edu.cn

${ }^{2}$ Collaborative Innovation Center for the Characteristic Resources Exploitation

of Dabie Mountains, Key Laboratories of Economic Forest Germplasm

Improvement and Comprehensive Resources Utilization of Hubei province,

College of Biology and Agricultural Resource, Huanggang Normal University,

Huanggang 438000, China

Full list of author information is available at the end of the article
} 


\section{Background}

Genomic imprinting is a universal epigenetic phenomenon evolved independently in animals and plants, which results in the biased expression of mono allele dependent on their parent-of-origin. In animals, a subset of imprinted genes have been identified and considered to be involved in the regulation of nutrient transfer from maternal tissue to embryo for embryo development (Frost and Moore 2010; Reik et al. 2003; Tycko and Morison 2002; Wood and Oakey 2006). In plants, although several imprinting genes have been identified in early embryos, genomic imprinting are primarily confined to the triploid endosperm, a transient tissue nourishing the developing embryo as placenta in animals. A series of imprinting genes have been identified in endosperms from Arabidopsis thaliana, Oryza sativa and Zea maize through a genome-wide survey (Guo et al. 2003; Hsieh et al. 2011; Luo et al. 2011; Zhang et al. 2011b). Among them, Polycomb Group (PcG) genes were explored more intensively due to their important roles in early endosperm development (Hermon et al. 2007; Ingouff et al. 2005; Kinoshita et al. 1999; Nallamilli et al. 2013; Yadegari et al. 2000).

In $A$. thaliana, PcG genes were shown to play critical roles in several important transition phases (e.g. from gametophyte to sporophyte), coordinating the development of endosperm, embryo proper and surrounded maternal tissues. Among them, two core components of PRC2 complex, MEA and FIS2, were revealed as imprinted genes and only transcribed from the maternal allele in endosperm (Kinoshita et al. 1999; Luo et al. 2000). The SET domain of MEA interacts directly with FIE to form a PcG complex, which is crucial for endosperm formation by controlling the activity of a number of imprinted genes in the endosperm (Baroux et al. 2006; Kohler et al. 2005; Spillane et al. 2000; Wang et al. 2006).

In $O$. sativa, great attention has also been paid on the PcG genes in endosperm due to the contribution of endosperm to the quality and yield of rice production. Six PcG genes including OsFIE1, OsFIE2, OsEMF2a, OsEMF2b, OsCLF, OsiEz1 have been identified according to the sequence similarity to known $P c G$ genes (Luo et al. 2009). The transcripts of all $P c G$ genes could be detected in endosperms. Among them, OsFIE1 and OsFIE2 were shown to play critical roles in endosperm development (Folsom et al. 2014; Li et al. 2014; Nallamilli et al. 2013). Overexpression of OSFIE1 result in the precocious cellularization and reduced seed size, whereas down regulation of OSFIE1 expression resulted in the reduced fertility and delayed embryo development (Folsom et al. 2014). OsFIE2 has also been reported to play essential roles in the regulation of rice vegetative and reproductive development, especially in the endosperm development and grain filling (Li et al. 2014).
However, whether these developmental defects in endosperm are attributed to the imprinting effect of $P c G$ genes is still largely unknown. OsFIE1 was shown as a maternally expressed imprinting gene in 5 days after pollination (dap) hybrid endosperms from the cross between Nipponbare and IR64 (Luo et al. 2009). And both maternal and paternal transcripts of other five $P c G$ genes (OsiEZ1, OsCLF, OsEMF2a, OsEMF2b, and OsFIE2) in 5 dap hybrid endosperms could be detected simultaneously (Luo et al. 2009). Since several elaborated events of endosperm development such as the formation of primary endosperm nucleus, the initiation of primary endosperm nucleus division and the onset of endosperm cellularization usually occur in the early stage before 5 dap (Brown et al. 1996), and the expression of imprinted genes may be developmental-stage-dependent, it is necessary to screen and confirm the imprinting pattern of these PcG genes in very early stages of endosperm development to figure out the role of the imprinted $P c G$ genes in seed development. Here, the imprinting pattern of $P c G$ genes in the entire process of endosperm development, especially in the early stages of endosperm development (within 3 dap), were analyzed. In addition, the influences of parental genetic background, alterative splicing form on the expression of parental alleles were also discussed in the present study.

\section{Results}

Identification of DNA polymorphisms of PcG genes among Nipponbare, 9311 and Zhonghua 11

To explore which allele of $P c G$ gene in endosperm was transcribed, the expression pattern of each $P c G$ gene in endosperms at different stages (from 1 dap to 7 dap) were carefully examined. The endosperm at different stages were isolated according to our previous method (Kuang et al. 2015). cDNA was prepared using mRNA extracted from endosperm at different stages. RT-PCR analysis results revealed that the transcripts of all $P c G$ genes could be detected in endosperms from 1 to 7 dap (Detection results from 3 dap endosperm shown in Fig. 1), giving a possibility to elucidate the dynamics of the expression of parental alleles in endosperms at different stages.

To distinguish two alleles of PcG genes in the genome, the DNA polymorphisms among three cultivated species including Nipponbare (Nip), 9311 and Zhonghua 11 (Zh11) were investigated firstly. The genomic sequences of each PcG gene were confirmed by the specific PCR amplification and subsequent Sanger sequencing. Comparing the PcG gene sequences from Nip, 9311 and Zh11, the single nucleotide polymorphisms (SNPs) were identified (Fig. 2 and Table 1). There is at least one base polymorphism located in the exon of each $P c G$ gene 
between two subspecies, which could be used to distinguish parental transcript efficiently.

\section{OsFIE1 is a maternally expressed imprinting gene at 7 DAP endosperm}

Early allelic specific expression pattern analysis $P c G$ genes in rice endosperm revealed that only OsFIE1 is maternally expressed imprinting gene among six $P c G$ genes (Luo et al. 2009). To confirm allelic expression patterns of $P c G$ genes in endosperm, three independent 7 dap hybrid endosperm cDNAs by crossing the Nip and the 9311 reciprocally were produced. Each cDNA sample from hybrid endosperm was subsequently used to amplify the SNP-containing sequence of each PcG transcript by RT-PCR with primer sequence flanking SNP. The PCR products were sequenced by Sanger sequencing and assessed for their parent-of-origin biased expression according to SNPs. Then, allele-specific expression analyses of $P c G$ genes in endosperm were performed as shown in Fig. 3. Among six PcG genes, only OsFIE1 showed monoallelic expression, confirming that OsFIE1 is a maternally expressed imprinting gene (Luo et al. 2009). Four PcG genes show biallelic expression in 7 dap endosperm, while OsEMF2a display either maternal expression pattern or a biallelic expression pattern depending on the direction of the cross between Nip and 9311.

\section{Dynamics of allele-specific expression of PCG genes} during endosperm development

Early researches indicated that several key endosperm developmental events including the onset of primary endosperm nucleus division, the compartmentalization of endosperm nuclei and the initiation of endosperm cellularization occur within 3 dap (Brown et al. 1996; Kuang et al. 2015). In addition, the effect of imprinting gene on endosperm development in A. thaliana is majorly on early endosperm development (Baroux et al. 2006; Jullien et al. 2006; Luo et al. 2000; Yadegari et al. 2000). Hence, to analyze allelic expression patterns of PcG genes comprehensively, hybrid endosperms were isolated at six key stages of endosperm development including stage 1 (1 dap), stage 2 (2 dap), stage 3 (3 dap), stage 4 (7 dap), stage 5 (10 dap) and stage 6 (15 dap), and cDNA samples from hybrid endosperms were prepared according to the previous protocol (Kuang et al. 2015).

Allele-specific expression analyses of $P c G$ genes in the process of endosperm development revealed that the expression pattern of two alleles of $P c G$ genes show dynamic changes as endosperm development, especially within 3 dap. Among six PcG genes, only OsCLF display a stable expression pattern, both transcripts from paternal allele and maternal allele could be detected in endosperms at six stages, indicating $O s C L F$ is not an imprinting gene in rice endosperm at these stages tested (Fig. 4). However,

Table 1 SNPs used to distinguish paternal and maternal transcripts in endosperm

\begin{tabular}{|c|c|c|}
\hline Name & SNP & Primers \\
\hline \multirow{2}{*}{ FIE1 } & \multirow{2}{*}{ TTTGAGATGATTTA[C]TGGTTCCT } & 5'-САТСТСТСТTCСТCATCACCG-3' \\
\hline & & 5'-CTTCCCTCAGTGTGCTTGTTG-3' \\
\hline \multirow{2}{*}{ FIE2 } & \multirow{2}{*}{ GCAAATAGGATA[G]CTGCTTCTGTT } & 5'- GACAATGAAATTGTGCTGTGGGAG-3' \\
\hline & & 5'- GCCATGCAACTTTGGTAACAAAGC-3' \\
\hline \multirow{2}{*}{ OsEMF2a(l) } & \multirow{2}{*}{ GCACATCTGGTCT[C]GAAGACCT } & 5'-TACATTCTGCCCTGCTGGATCTG-3' \\
\hline & & 5'-GAAGGCATCTTTGAAGATAAGGAGG-3' \\
\hline \multirow{2}{*}{$O s E M F 2 a(s)$} & \multirow{2}{*}{ GCACATCTGGTCT[C]GAAGACCT } & 5'-CTTGACCTAGGCTGGAATACCG-3' \\
\hline & & 5'CAGATGAGTACACATTTATAGCCAC-3' \\
\hline \multirow{2}{*}{$O s E M F 2 b$} & \multirow{2}{*}{ AATTGATGTTACT[A]CATAACTGT } & 5'-CATTGGAGCAAGTTTTCTCAGATCG-3' \\
\hline & & 5'-CAGTTTGGTCTTGATACTGCTCTAC-3' \\
\hline \multirow{2}{*}{ OsCLF } & \multirow{2}{*}{ CAATCACT[C]TTCCAGGACCTGC } & 5'-CTCTAAACCAGATGCTATCATGGAG-3' \\
\hline & & 5'-CACAAGGAGTGTATTGTCGACAAG-3' \\
\hline \multirow{2}{*}{ OsiEZ1(l) } & \multirow{2}{*}{ CGAGAAGTACTGC $[$ T]GGGTATGT } & 5'-CTGGAAGCACAACTAACACTACTG-3' \\
\hline & & 5'-CACGCTTATCTGCTTCTCTATGAG-3' \\
\hline \multirow{2}{*}{ OsiEZ1(s) } & \multirow{2}{*}{ CGAGAAGTACTGC $[$ T]GGGTATGT } & 5'-AGCATGGGTATTGCCTTGAATACG-3' \\
\hline & & 5'-CCAGTGACACAATTATTTACTCATCTC-3' \\
\hline
\end{tabular}

SNP in each transcript was labelled with boldface character inside and outside of square brackets in red colour 


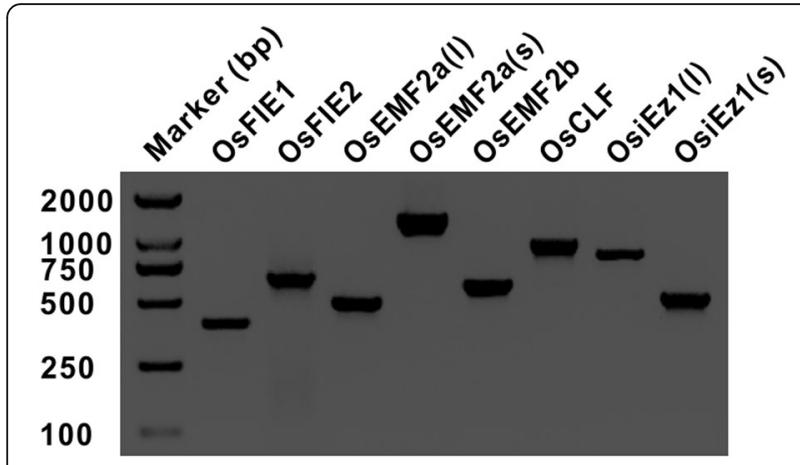

Fig. 1 The expression of PCG genes in endosperm confirmed by RTPCR. Distinction between OsEMF2a(l) and OsEMF2a(s) based on different amplification primers and sequencing primers (See Additional file 1: Table S1)

parent alleles of other five $P c G$ genes show diverse transcriptional activities in endosperms at different stages, especially in stage 1 and stage 2 , demonstrating a stagespecific manner. At stage 1, only maternal allele of four PcG genes (OsFIE2, OsEMF2a, OsEMF2b and OsiEZ1) had been transcribed its transcripts.

In more details, maternal allele of OSFIE1 was always expressed in endosperm from 1 to 15 dap in Nip $\times 9311$.
By contrast, paternal allele of OsFIE1 was only expressed at 1 dap endosperm in $9311 \times$ Nip. Maternal allele of OsFIE2 was first expressed in 1 dap endosperm and then parental alleles of OsFIE2 were both expressed in endosperm at following stages in both Nip $\times 9311$ and $9311 \times$ Nip. More interestingly, OsEMF2a was maternally expressed in early and late endosperm, while paternal allele of OsEMF2a were only expressed in the middle stage in Zh11 × 9311. With similar expression pattern as OsFIE2, both $O s E M F 2 b$ and $O S i E Z 1$ were maternally expressed in early endosperm and were parentally expressed in following stages. All these data implied that endosperm at free nucleus stage is critical for parent-of-origin gene analysis in rice and the expression of the imprinted genes are highly dynamic or stage-dependent.

\section{The imprinting pattern of PcG genes are influenced by genetic background}

As mentioned above, parent alleles of $P c G$ genes display different transcriptional activities based on the direction of the cross between Nip and the 9311, suggesting that the imprinting pattern of $P c G$ genes are influenced by different parent combinations. To address this hypothesis, the imprinting pattern of $P c G$ genes in two
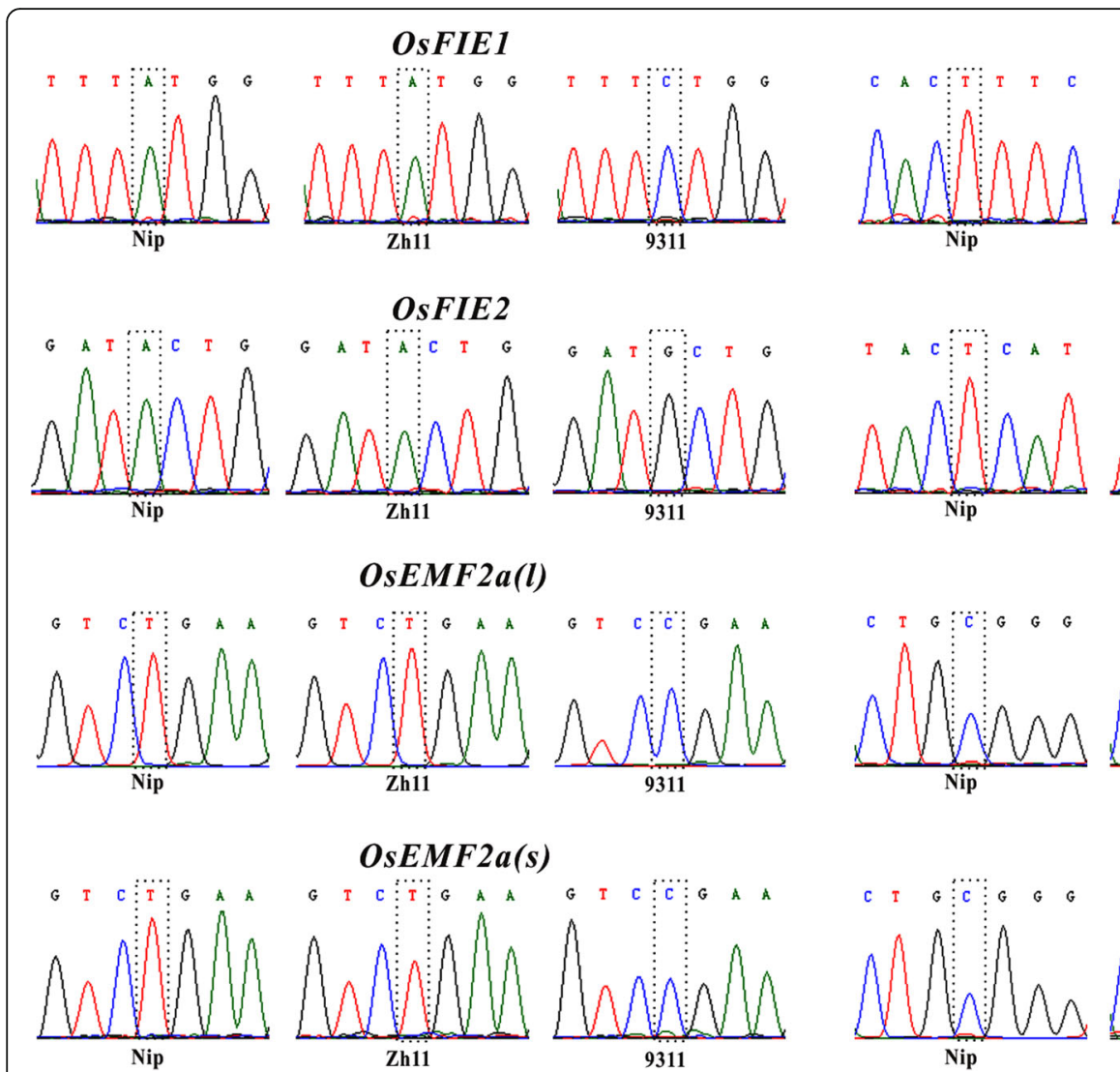

OsCLF
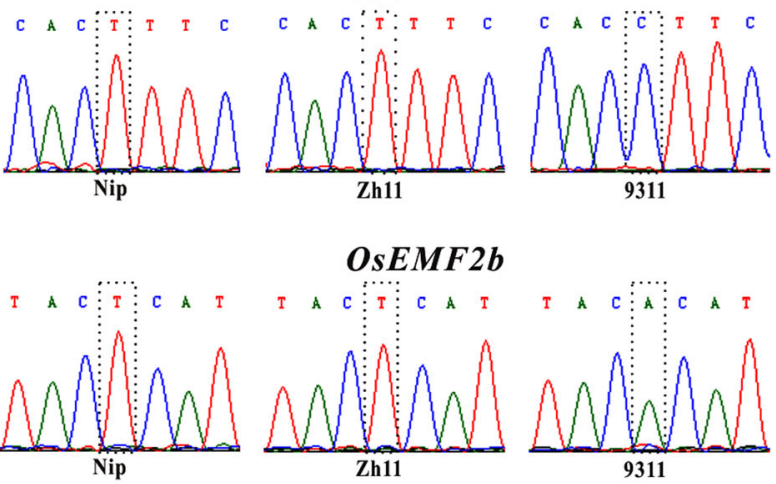

OsEMF2b

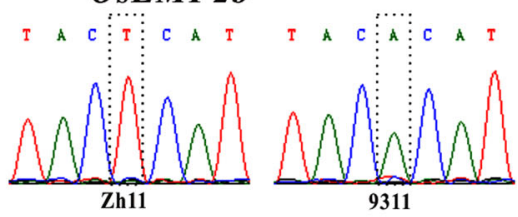

OsiEz1(l)
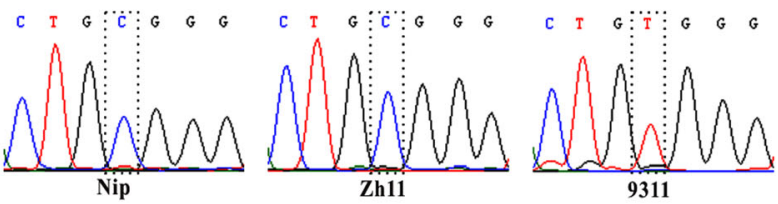

OsiEz1(s)
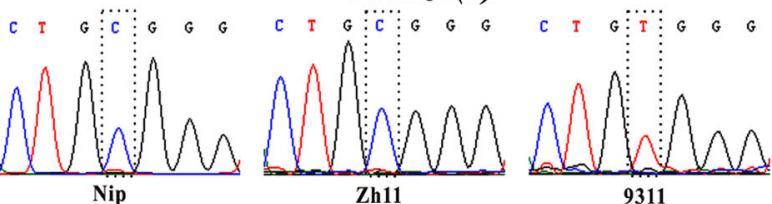

Fig. 2 Identification of single nucleotide polymorphisms (SNPs) of PCG genes among Nip, 9311 and Zh11. SNPs for each transcript was labeled with dashed box 


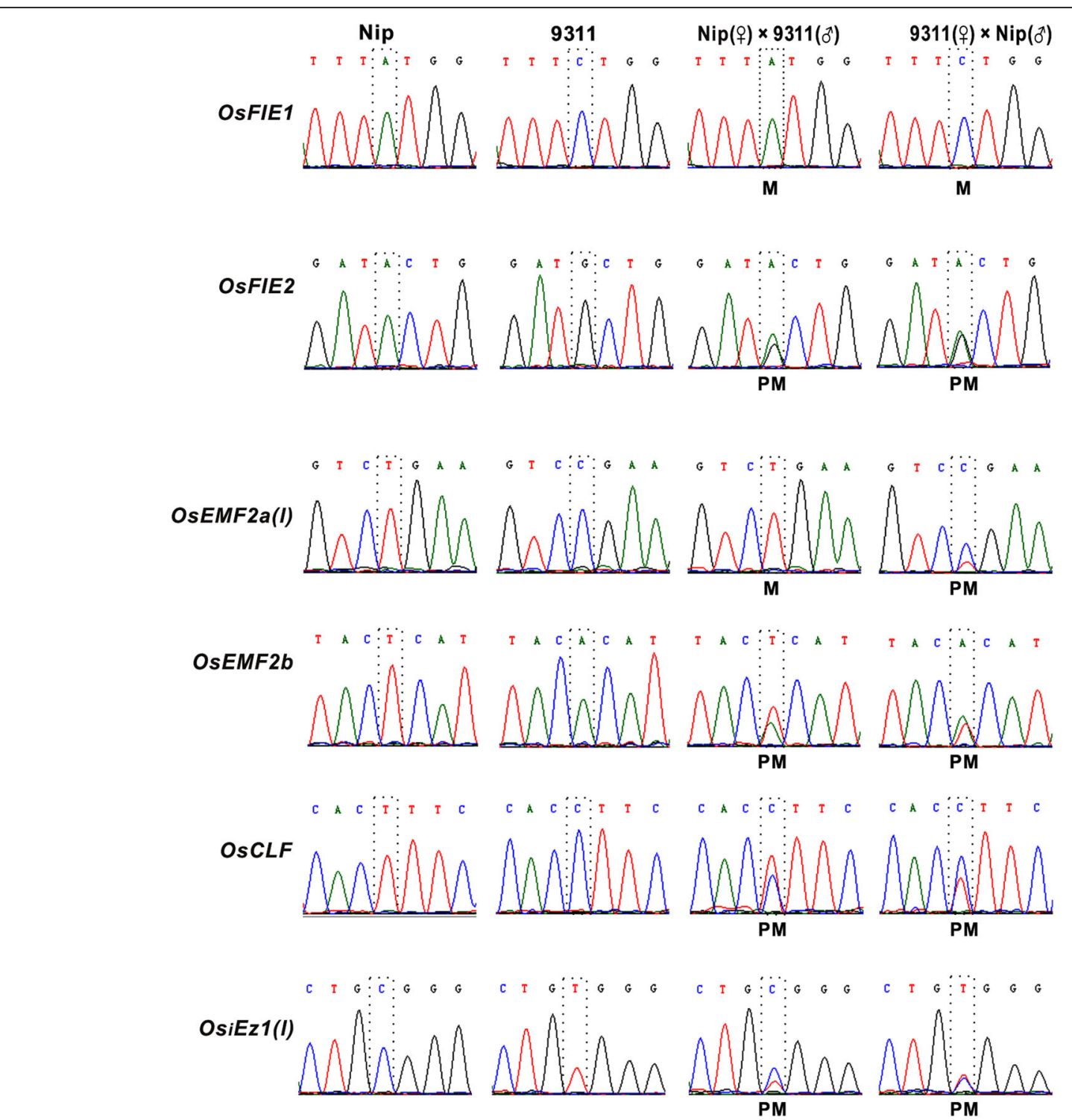

Fig. 3 OSFIE1 is a maternally expressed imprinting gene at 7 DAP endosperm. SNPs for each transcript was labeled with dashed box. Each data has been confirmed by three independent experiments

reciprocal cross combinations (Nip or Zh11 with 9311) was compared. The results revealed that the $P c G$ genes exhibit notable variation in parental allele activation in the process of endosperm development and the transcriptional activities of two alleles of $P c G$ genes are different dependent on the combination of two parents. Among six PcG genes, OsCLF showed a consistent bi-allelic expression pattern in endosperms in two independent reciprocal cross combinations. Maternal allele of OsEMF2b was firstly activated in endosperms (within 2 dap), and parental allele was activated in 3 dap endosperm subsequently. However, other $P c G$ genes display notable variation in parental allele activation in two different reciprocal cross combinations. For example, only OsiEZ1 transcript transcribed from the maternal allele could be detected in 3 dap hybrid endosperm derived from the cross between Nip and 9311, but the transcripts both from maternal allele and paternal allele could be detected simultaneously in 3 dap hybrid endosperm derived from the cross between Zh11 and 9311 (Fig. 5). In addition, the maternal allele of OsFIE1 was expressed at 1 dap in Zh11 $\times 9311$, while paternal allele of OSFIE1 was also expressed in 1 dap endosperm $9311 \times$ Zh11. Similar expression pattern of OsFIE2 is also observed at 1 dap in both Zh11 $\times 9311$ and $9311 \times \mathrm{Zh} 11$. Interestingly, in Nip $\times 9311$ reciprocal crosses, it was maternal allele of OsFIE2 expressed at 1 dap, whereas, in Zh11 $\times 9311$ reciprocal crosses OsFIE2 seems a dominant allele at 1 dap and associated with 

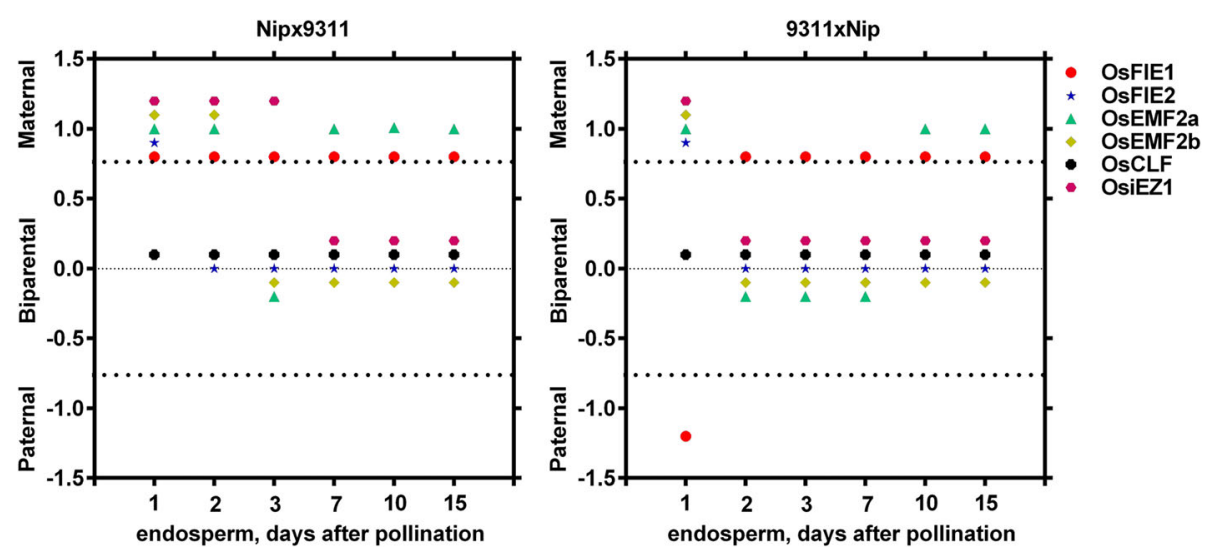

Fig. 4 Dynamics of allele-specific expression of PCG genes during endosperm development. The dots above the first dash line indicated only transcripts derived from maternal allele could be detected. The dots nearby the middle dash line indicated both transcripts from paternal allele and maternal allele could be detected simultaneously. The dots below the third dash line indicated only transcripts derived from paternal allele could be detected. Each data has been confirmed by three independent experiments

Zh11 whenever Zh11 was used as paternal or maternal cultivar. These results indicated the expression patterns of certain gene could be varied by crossing with different parental cultivars and this dominant expression of the imprinted genes associated with specific cultivar is also stage-dependent.

\section{Different alternative splicing forms of PcG genes display different imprinting pattern}

Alternative splicing is an important gene regulatory mechanism in eukaryotes, which results in a single gene coding for multiple proteins. In plants, about least $20 \%$ multi-exon genes are alternatively spliced (Estrada et al. 2015). However, whether the two alleles of different alternative splicing forms display similar imprinting pattern is still largely unknown. To address this question, two representative PcG genes (OsEMF2a and OsiEZ1) with distinguishable splicing forms were selected to analyze the imprinting pattern of different splicing forms. OsEMF2a and OsiEZ1 could generate different splicing isoforms with different size. In order to distinguish alternative splicing forms more efficiently, two representative splicing forms, the longest $(l)$ and the shortest $(s)$ transcripts of OsEMF2 $a$ and OsiEZ1, were selected to analyze the expression of two alleles (Fig. 2 and Table 1). The results revealed that OsiEZ1(l) and OsiEZ1(s), two alternative splicing forms OsiEZ1 displayed the same allele-specific expression pattern in the 3 dap endosperm from reciprocal crosses between Nip and 93-11 (Fig. 6). However, two isoforms of OsEMF2a display different parent-of-origin expression pattern in the 3 dap endosperm (Fig. 6), providing a clear example of parent of origin-biased transcript isoforms arising from the same gene.

\section{Discussion}

Uniparental expression pattern of PCG genes is developmental stage-dependent during endosperm formation

One clear finding in the present study is that uniparental expression pattern of $P c G$ genes displays a developmental stage-dependent manner. OsFIE1 is only a maternally expressed imprinting gene in the whole process of hybrid endosperm $(\mathrm{Nip} \times 9311)$ development, and no biased expression of OsCLF alleles was found in any developmental stages of hybrid endosperm (Nip $\times 9311)$. However, the activation or silencing of two alleles of other four $P c G$ genes shows dynamic changes at different stages of endosperm development, suggesting that the expression pattern of parental-origin genes changes as endosperm development, especially in early stages of endosperm development. Similar stage-specific manner of parentalorigin genes has also been reported in hybrid embryos. In tobacco, only transcripts from paternal allele of EB426694 and CN744644 could be detected in hybrid zygote, whereas both paternal and maternal transcripts could be detected simultaneously in eight-celled embryo (Zhang et al. 2011a). In maize, allele-specific expression assays of 90 genes in endosperms at different stages revealed that only eight of them exhibited persistent maternally or paternally biased expression at multiple stages of endosperm development (Stupar et al. 2007). In A. thaliana, only paternal allele of FUSCA3 was activated at the 2-4 cell embryo stage, but either maternal or biallelic was activated at the globular embryo stage depending on the direction of the cross between Columbia-0 (Col-0) and Landsbergerecta (Ler) (Raissig et al. 2013). These data revealed that expression pattern of allele specific genes both in embryo and endosperm display a developmental stage-dependent manner, and thus parental and maternal transcripts may 

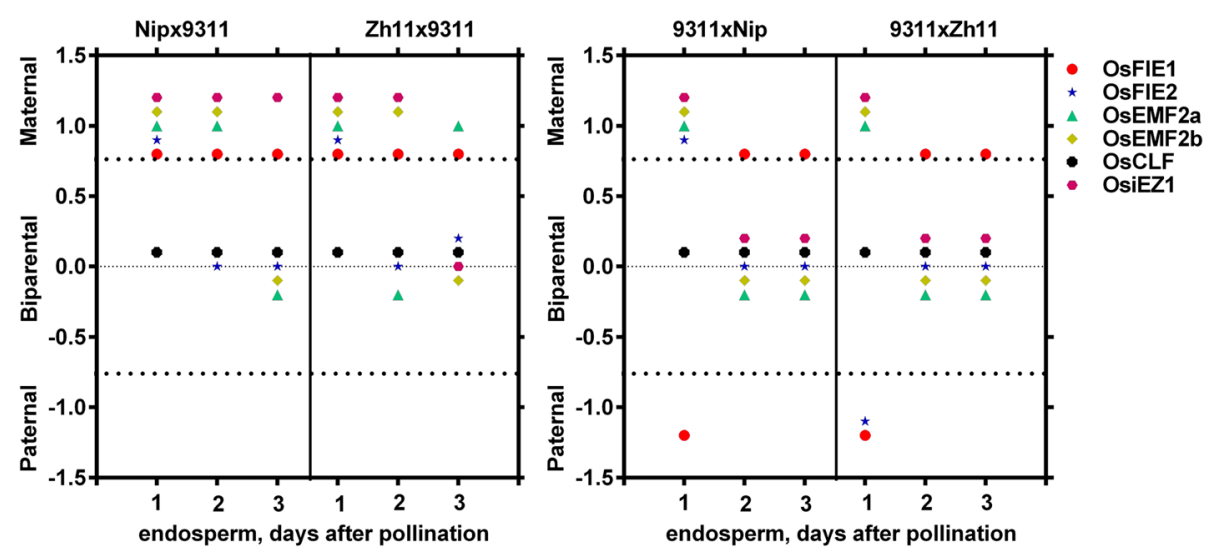

Fig. 5 Influence of genetic background on allele-specific expression of PCG genes. The dots above the first dash line indicated only transcripts derived from maternal allele could be detected. The dots nearby the middle dash line indicated both transcripts from paternal allele and maternal allele could be detected simultaneously. The dots below the third dash line indicated only transcripts derived from paternal allele could be detected. Each data has been confirmed by three independent experiments

have specific contribution to the embryo or endosperm development at specific stage.

Endosperm development in rice experience several critical early stages, including the fusion of the sperm cell with central cell, first cell division of primary endosperm nucleus or promotion of endosperm development, free nuclear movement and oriented distribution, the initialization of cellularization. The molecular mechanism to regulate these critical stages are not well understood. We speculate that there was a correlation between gene imprinting and these critical developmental events. Different imprinted genes may involve in a specific developmental event, not all these events. In addition, parents may differentially contribute to the same developmental events. That's probably why their expression is highly dynamic and in a stage-dependent manner.

\section{Alternative splicing forms of a certain transcript displays different expression pattern}

Another interesting question raised in the present study is that alternative splicing forms of $P c G$ genes display different imprinting patterns. The imprinting pattern of two splicing forms of OsEMF2a and OsiEZ1 were compared in the experiment. Most obviously, the short transcript for OsEMF2a (AK069556) is uniparental expressed, but the long transcript (AK120470) is biparental expressed in hybrid endosperm. A similar phenomenon has also been found in inter-subspecies hybrid mice. Allele-specific polyadenylation sites were found at a novel murine imprinted gene (H13). Maternal allele preferentially produces the long transcripts, whereas the truncated transcripts are preferentially originated from paternally derived alleles (Wood et al. 2008). This indicates the different roles of the same gene as biparental expressed gene or imprinted genes. Different parentally biased isoforms could generate from same gene via alternative splicing, providing a new aspect of genomic imprinting. The same gene could play both imprinted and nonimprinted roles by produce different transcripts. In this case, it is difficult to name it as an imprinted gene or nonimprinted gene, probably, to be more accurate, we may describe it as imprinted transcript or non-imprinted transcript of the gene. This finding enhances our understanding of the complexity of genomic imprinting.

\section{Uniparental expression pattern of PCG genes is influenced by parental genetic background}

It was reported that it will suffer a very important effects to the offspring's phenotypic and quality traits by using different male and female parents as hybrid material, or exchanging parents' position for reciprocal cross. This phenomenon is usually considered to be the result from unbalanced dose ratio between maternal genome and paternal genome during the reciprocal-crossing processes (Dilkes et al. 2008; Scott et al. 1998). Our work provides new explanations for the phenomenon. The uniparental expression pattern of genes is obviously influenced by parental genetic background. Some imprinted genes of certain cultivars could predominantly expresses in the hybrids whenever the cultivar is used as maternal or paternal material in the crosses, indicating that the behavior of the imprinted genes could be associated with certain genotype. The regulatory mechanism underlying the phenomenon is interesting but not yet understood. However, it is clear that the imprinting effects of the genes will certainly influence the phenotype, e.g. endosperm development, therefore, it may enable some specific cultivar with superiority to control specific phenotypes or developmental characters in hybrids. Thus, it might be an alternative explanation for the parent-associated 


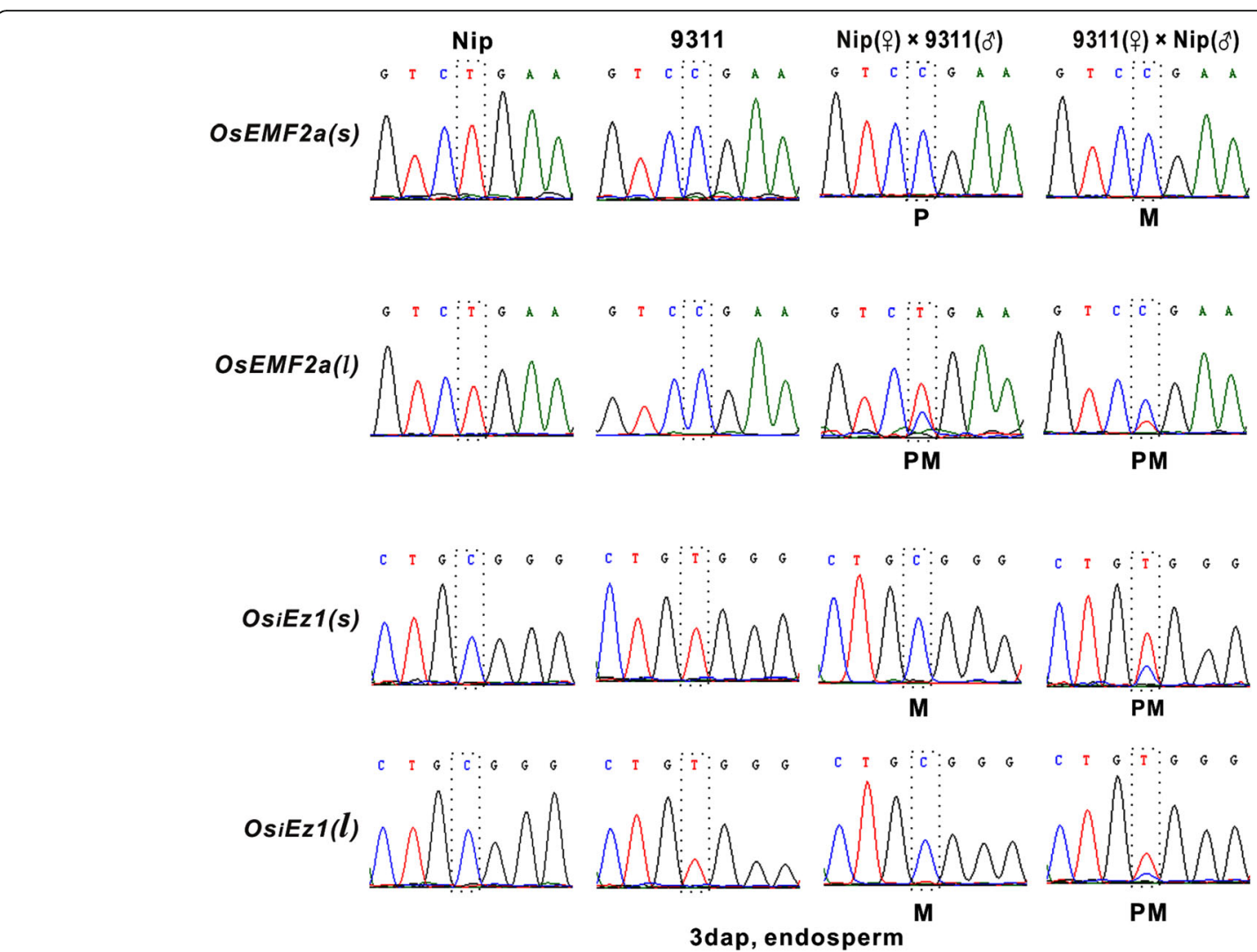

Fig. 6 Different alternative splicing form of PCG genes display different imprinting pattern. SNPs for each transcript was labeled with dashed box. Each data has been confirmed by three independent experiments

characters in crop crosses and provides new clue for the selection of the parents in crop breeding.

\section{Methods}

\section{Plant materials}

Three Oryza sativa species including two Japonica cultivars Nipponbare, Zhonghua 11 and one Indica cultivar 9311 were used in the present study, which were cultivated in the greenhouse with $13 \mathrm{~h}$ of illumination every day. The daytime temperature was $30^{\circ} \mathrm{C}$, and the night temperature was $25^{\circ} \mathrm{C}$.

\section{Polymorphism detection between rice species}

In order to detect DNA polymorphisms among Nip, 9311 and Zh11, the genomic sequences of PcG genes (OsFIE1, OsFIE2, OsEMF2a, OsEMF2b, OsCLF, OsiEz1) were downloaded from rice genomic database, which is from "http://www.ricedata.cn/gene/" or "https://rapdb.dna.affrc. go.jp/" in this experiment. In the meantime the sequences of $P c G$ genes from Nip, 9311 and Zh11 were confirmed through PCR, respectively. And the genomic sequences of $P c G$ genes from two different species were aligned and compared to detect DNA polymorphisms.

\section{RNA extraction and RT-PCR}

The collection of hybrid ovaries at the same stages, endosperm isolation and mRNA extraction were carried out according to the previous protocol (Kuang et al. 2015). For the crosses, the flowers were previously labeled, emasculated, and hand-pollinated. After emasculation and pollination, the flowers were covered by paper bag, therefore, self-pollination was totally excluded and the time after pollination is exactly controlled. The detection on SNP loci in OsCLF ensured the hybrid ovaries for the experiments. cDNA were synthesized using SuperScript III Reverse Transcriptase (Thermo fisher Scientific) under the conditions recommended by the manufacturer. RTPCR was performed in a $20 \mu \mathrm{l}$ PCR mixture containing $2 \mu \mathrm{l}$ of 10× Ex Taq buffer (including $\mathrm{Mg}^{2+}$ ), $200 \mu \mathrm{M}$ dNTPs, $0.2 \mu \mathrm{M}$ of primers, $0.5 \mathrm{U}$ Ex Taq DNA polymerase (Takara), and cDNA prepared from endosperms at different stages. PCR conditions are performed as follows: initial denaturation at $94{ }^{\circ} \mathrm{C}$ for $2 \mathrm{~min} ; 40$ amplification cycles with denaturation at $94^{\circ} \mathrm{C}$ for $30 \mathrm{~s}$, annealing at $56^{\circ} \mathrm{C}$ for $30 \mathrm{~s}$; extension at $72^{\circ} \mathrm{C}$ for $1 \mathrm{~min}$; and a final incubation at $72{ }^{\circ} \mathrm{C}$ for $5 \mathrm{~min}$. Each PCR products were purified and sequenced to distinguish which allele was transcribed according to DNA polymorphisms. 
For genes with multiple transcripts such as OsEMF2a, we designed primers on the specific sequences of different regions from different transcripts, which can amplify specific splices of different lengths (Table 1). In general, long transcripts can translate proteins, and short transcripts cannot translate proteins without initiation codon ATG.

\section{Additional file}

Additional file 1: Table S1. Primers of Distinction between OsEMF2a(l) and OsEMF2a(s). (DOC $27 \mathrm{~kb}$ )

\section{Abbreviations}

9311: Oryza sativa subsp. indica (9311); DAP/dap: Days after pollination; Nip: Oryza sativa subsp. japonica (Nipponbare); PcG: Polycomb group; PCR: polymerase chain reaction; PRC2: Polycomb Repressive Complex 2; RTPCR: Reverse transcriptase polymerase chain reaction; SNPs: Single nucleotide polymorphisms; Zh11: Oryza sativa subsp. japonica (Zhonghua 11)

\section{Acknowledgements}

The author acknowledge the use of the facilities, and scientific and technical assistance of the State Key Laboratory of hybrid rice in Wuhan University of China. The author also thank Prof. Mengxiang Sun and Dr. Peng Zhao for constructive comments on the manuscript.

\section{Authors' contributions}

QK conducted experiment; YHW analyzed the images and data; QK and SSL designed all experiments and wrote the manuscript. All authors read and approved the final manuscript.

\section{Funding}

The research reported in this publication was supported by funding from doctoral start-up fund and "11531" engineering construction projects of Nanchang Normal University. The work was financially supported by the National Natural Science Foundation of China (31430007) and "973 Program" (2013CB126900).

\section{Availability of data and materials}

The datasets supporting the conclusions of this article are included within the article.

\section{Ethics approval and consent to participate}

Not applicable.

\section{Consent for publication}

Not applicable.

\section{Competing interests}

The authors declare that they have no competing interests.

\section{Author details}

'Department of Biology, Institute of Biotechnology, Nanchang Normal College, Nanchang 330032, China. ${ }^{2}$ Collaborative Innovation Center for the Characteristic Resources Exploitation of Dabie Mountains, Key Laboratories of Economic Forest Germplasm Improvement and Comprehensive Resources Utilization of Hubei province, College of Biology and Agricultural Resource, Huanggang Normal University, Huanggang 438000, China. ${ }^{3}$ College of Software, East China Jiao Tong University, Nanchang 330013, China.

\section{Received: 25 February 2019 Accepted: 27 June 2019}

\section{Published online: 13 August 2019}

\section{References}

Baroux U, Gagliardini V, Page DR, Grossniklaus U (2006) Dynamic regulatory interactions of Polycomb group genes: MEDEA autoregulation is required for imprinted gene expression in Arabidopsis. Genes Dev 20:1081-1086

Brown RC, Lemmon BE, Olsen OA (1996) Development of the endosperm in rice (Oryza sativa L): Cellularization. J Plant Res 109:301-313
Dilkes BP, Spielman M, Weizbauer R, Watson B, Burkart-Waco D, Scott RJ, Comai L (2008) The maternally expressed WRKY transcription factor TTG2 controls lethality in interploidy crosses of Arabidopsis. PLoS Biol 6:2707-2720

Estrada AD, Freese NH, Blakley IC, Loraine AE (2015) Analysis of pollenspecific alternative splicing in Arabidopsis thaliana via semi-quantitative PCR. PeerJ 3:e919

Folsom JJ, Begcy K, Hao X, Wang D, Walia H (2014) Rice fertilization-independent Endosperm 1 regulates seed size under heat stress by controlling early endosperm development. Plant Physiol 165:238-248

Frost JM, Moore GE (2010) The importance of imprinting in the human placenta. PLOS Genet 6:e1001015

Guo M, Rupe MA, Danilevskaya ON, Yang X, Hu Z (2003) Genome-wide mRNA profiling reveals heterochronic allelic variation and a new imprinted gene in hybrid maize endosperm. Plant J 36:30-44

Hermon P, Srilunchang KO, Zou J, Dresselhaus T, Danilevskaya ON (2007) Activation of the imprinted Polycomb group Fie1 gene in maize endosperm requires demethylation of the maternal allele. Plant Mol Biol 64:387-395

Hsieh TF, Shin JY, Uzawa R, Silva P, Cohen S, Bauer MJ, Hashimoto M, Kirkbride RC, Harada JJ, Zilberman D, Fischer RL (2011) Regulation of imprinted gene expression in Arabidopsis endosperm. Proc Natl Acad Sci U S A 108:1755-1762

Ingouff M, Haseloff J, Berger F (2005) Polycomb group genes control developmental timing of endosperm. Plant J 42:663-674

Jullien PE, Katz A, Oliva M, Ohad N, Berger F (2006) Polycomb group complexes self-regulate imprinting of the Polycomb group gene MEDEA in Arabidopsis. Curr Biol 16:486-492

Kinoshita T, Yadegari R, Harada JJ, Goldberg RB, Fischer RL (1999) Imprinting of the MEDEA polycomb gene in the Arabidopsis endosperm. Plant Cell 11:1945-1952

Kohler C, Page DR, Gagliardini V, Grossniklaus U (2005) The Arabidopsis thaliana MEDEA Polycomb group protein controls expression of PHERES1 by parental imprinting. Nat Genet 37:28-30

Kuang Q, Yu X, Peng X, Sun MX (2015) The isolation of early nuclear endosperm of Oryza sativa to facilitate gene expression analysis and screening imprinted genes. Plant Methods 11:49

Li S, Zhou B, Peng X, Kuang Q, Huang X, Yao J, Du B, Sun MX (2014) OsFIE2 plays an essential role in the regulation of rice vegetative and reproductive development. New Phytol 201:66-79

Luo M, Bilodeau P, Dennis ES, Peacock WJ, Chaudhury A (2000) Expression and parent-of-origin effects for FIS2, MEA, and FIE in the endosperm and embryo of developing Arabidopsis seeds. Proc Natl Acad Sci U S A 97:10637-10642

Luo M, Platten D, Chaudhury A, Peacock WJ, Dennis ES (2009) Expression, imprinting, and evolution of rice homologs of the polycomb group genes. Mol Plant 2:711-723

Luo M, Taylor JM, Spriggs A, Zhang H, Wu X, Russell S, Singh M, Koltunow A (2011) A genome-wide survey of imprinted genes in rice seeds reveals imprinting primarily occurs in the endosperm. PLoS Genet 7:e1002125

Nallamilli BR, Zhang J, Mujahid H, Malone BM, Bridges SM, Peng Z (2013) Polycomb group gene OsFIE2 regulates rice (Oryza sativa) seed development and grain filling via a mechanism distinct from Arabidopsis. PLoS Genet 9:e1003322

Raissig MT, Bemer M, Baroux C, Grossniklaus U (2013) Genomic imprinting in the Arabidopsis embryo is partly regulated by PRC2. PLoS Genet 9: e1003862

Reik W, Constancia M, Fowden A, Anderson N, Dean W, Ferguson-Smith A, Tycko B, Sibley C (2003) Regulation of supply and demand for maternal nutrients in mammals by imprinted genes. J Physiol Lond 547:35-44

Scott RJ, Spielman M, Bailey J, Dickinson HG (1998) Parent-of-origin effects on seed development in Arabidopsis thaliana. Development 125:3329-3341

Spillane C, MacDougall C, Stock C, Kohler C, Vielle-Calzada JP, Nunes SM, Grossniklaus U, Goodrich J (2000) Interaction of the Arabidopsis Polycomb group proteins FIE and MEA mediates their common phenotypes. Curr Biol 10:1535-1538

Stupar RM, Hermanson PJ, Springer NM (2007) Nonadditive expression and parent-of-origin effects identified by microarray and allele-specific expression profiling of maize endosperm. Plant Physiol 145:411-425

Tycko B, Morison IM (2002) Physiological functions of imprinted genes. J Cell Physiol 192:245-258

Wang D, Tyson MD, Jackson SS, Yadegari R (2006) Partially redundant functions of two SET-domain polycomb-group proteins in controlling initiation of seed development in Arabidopsis. Proc Natl Acad Sci U S A 103:13244-13249 
Wood AJ, Oakey RJ (2006) Genomic imprinting in mammals: emerging themes and established theories. PLoS Genet 2:e147

Wood AJ, Schulz R, Woodfine K, Koltowska K, Beechey CV, Peters J, Bourc'his D, Oakey RJ (2008) Regulation of alternative polyadenylation by genomic imprinting. Genes Dev 22:1141-1146

Yadegari R, Kinoshita T, Lotan O, Cohen G, Katz A, Choi Y, Katz A, Nakashima K, Harada JJ, Goldberg RB, Fischer RL, Ohad N (2000) Mutations in the FIE and MEA genes that encode interacting polycomb proteins cause parent-of-origin effects on seed development by distinct mechanisms. Plant Cell 12:2367-2382

Zhang JE, Luo A, Xin HP, Zhao J, Li SS, Qu LH, Ma LG, Scholten S, Sun MX (2011a) Genes of both parental origins are differentially involved in early embryogenesis of a tobacco interspecies hybrid. PLoS One 6:e23153

Zhang M, Zhao H, Xie S, Chen J, Xu Y, Wang K, Zhao H, Guan H, Hu X, Jiao Y, Song W, Lai J (2011b) Extensive, clustered parental imprinting of proteincoding and noncoding RNAs in developing maize endosperm. Proc Natl Acad Sci U S A 108:20042-20047

\section{Publisher's Note}

Springer Nature remains neutral with regard to jurisdictional claims in published maps and institutional affiliations.

\section{Submit your manuscript to a SpringerOpen ${ }^{\circ}$ journal and benefit from:}

- Convenient online submission

- Rigorous peer review

- Open access: articles freely available online

High visibility within the field

- Retaining the copyright to your article

Submit your next manuscript at $\boldsymbol{\nabla}$ springeropen.com 